\title{
APPLICATIONS OF CONVERSATIONAL THINKING: THE ROLE OF COLLECTIVE ACTION IN MERGING CONTEXTS DOI: https://dx.doi.org/10.4314/ajct.v1i1.7
}

\author{
Submission: May 17, $2021 \quad$ Acceptance: October 1, 2021 \\ Leyla TAVERNARO-HAIDARIAN, \\ University of Johannesburg \\ Johannesburg, South Africa \\ Email: leylahaid@me.com
}

ORCID No: https://orcid.org/0000-0002-3860-4737

\begin{abstract}
Conversationalism is based on the idea that the truth of our propositions depends on the context in which they are asserted and describes a process of relational yet critical exchange between epistemic agents. However, experiences in applying the conversational method in a micro intercultural setting show that when individuals who are engaged in this creative struggle take collective action together their contexts may in fact converge, thereby frustrating a continuous collision of theses. As a point of departure for this submission, I take an auto-ethnographic approach and share my reflections on a series of conversational encounters between proponents of two traditions who were faced with the challenge of practically collaborating on an educational policy. I then draw on discourse theory to discuss the role of this joint action in producing contextual overlap and theoretical nearness without thwarting the goal of epistemic sophistication, detailing some theoretical as well as practical implications.
\end{abstract}

Keywords: conversationalism, philosophy, discourse, migration, culture, collective action

\section{Introduction}

Conversational philosophy thrives on a respectful yet rigorous exchange between proponents of various theses (CHIMAKONAM $2015 ; 2017 \mathrm{ab})$. Probing the validity of each thesis from within its specific thought world or context is key. The goal of a conversational 
exchange, therefore, is not for viewpoints to synthesize or come together but rather to allow each proposition to gain sophistication within its own specific context and through a constant, relational process in which epistemic agents challenge and refine each other's views (CHIMAKONAM 2021). However, my experiences of applying the conversational method in a micro-intercultural setting reveal that when individuals who are engaged in this 'creative struggle' (CHIMAKONAM 2015; 2017a \& b; 2021) take collective action together their contexts or thought worlds may converge. This can frustrate a collision of theses, and yet not necessarily bar them from becoming more sophisticated. In order to illustrate this, I share an auto-ethnographic narrative of my experiences with the conversational method in an intercultural setting in Austria. I do so from the vantage point of a communication scholar and community activist who was engaged in a series of encounters between two groups of youth with mixed experiential and cultural backgrounds. These groups explored what policy they would design in order to address how religious headscarves, or hijabs, should be handled in Austrian schools and community centers. Faced with the challenge of practically collaborating, they actioned a decision on this policy for their own community center and 'returned to the conversation' at a later stage in order to reflect on the experience and refine their epistemic positions. By drawing on discourse analysis, I discuss the significance of this joint action in producing contextual overlap and theoretical nearness without impeding the goal of epistemic sophistication. In other words, I propose that collective action, or a shared lived experience, was able to and can bring together thought worlds and realities (i.e. contexts), while, nonetheless, enriching the conversational process of creative struggle.

The implications of this for the theory development and research on conversationalism are varied. For example, it suggests that the relationship of contextual worlds, one to another, may follow a converging trajectory. Furthermore, while a critical interplay of clashing ideas is desirable in producing higher levels of epistemic refinement, overlap or even synthesis may not always be counterproductive. Here, the (Western liberal) dichotomy between absolutism and relativism or objectivism and subjectivism (see SMITH \& KARLBERG 2009, 60) can be reconsidered, allowing for the possibility of a higher order or objective truth towards which 
nested and contextual ones can gradually move. This advances the decolonial project of the conversational method, as it contributes to nuanced ideas of a "willful, creative and critical" relationalism (CHIMAKONAM 2017, 15). It also has practical implications for macro-intercultural engagements, such as those between Austrians (or Europeans) and their growing immigrant populations. It suggests that these various communities of identity can co-create culture through shared experiences.

\section{Aim \& Method}

My aim with this paper is to show that when a conversational exchange moves beyond the theoretical realm to include tangible and 'collective action' (CORREA 2020), contexts or thought worlds may converge. In other words, what existed in separate thought worlds is suddenly brought into a shared lived experience and may inadvertently cause epistemic closeness. I, therefore, discuss the significance of collective action in converging contexts and theses, even where no such intention is necessarily present.

For this, I take an auto-ethnographic approach and reflect on my participant observations of a series of conversational engagements between proponents of two religious/cultural traditions. Autoethnography seeks to describe and systematically analyze personal experience in order to understand cultural experience (ELLIS 2004). As a method, it is both a process and product (ELLIS, ADAMS \& BOCHNER 2011). As is the case in this paper, experiences are often gathered and reflected upon retrospectively, selectively and in combination with other texts in order to address the nexus between theory and practice (HERRMANN 2005). However, autoethnography is not mere storytelling. It requires a critical examination of purposively highlighted experiences in order to illustrate and better understand facets of cultural experience for theory development. I do this through discourse analysis.

An inclusive understanding of discourse analysis (USSHER \& PERZ 2019; WETHERELL 1998) allows me to focus on the action orientation of the conversations (or conversational experiences) I'm considering. In other words, I focus on what the conversation is doing, rather than what it means or what it is saying (see WILLIG 2008, 98). For this, I consider both the individual and interpersonal level of 
communication as well as the larger social context within which this experience takes place, including notions of broad cultural representation and power (FOUCAULT 1987). As a woman researcher with a complex ethico-cultural lens, I also consider the gendered dimension of discursive practice (GAVEY 2011), paying attention to the ways in which conversations about the hijab include or exclude female agency. However, these considerations are not foregrounded, as the purpose of this paper is to understand the theoretical implications for the conversational method rather than to trace the thematic evolution of specific discourses.

This auto-ethnographic analysis, then, pans out as a combination of purposive selection, narrative description and subsequent analysis, with a view to understanding how the process of conversational engagement and creative struggle is affected through collective action, thereby proposing new avenues for theory development and research. As such, I use conversationalism as a method of research in the real world while simultaneously investigating how it operates as my object of analysis. My contribution is limited (but also perhaps enriched) by my personal biases and my professional experience as a communication scholar with a very modest understanding of the field of philosophy.

\section{Key Theories}

Contributing thoughts on the applications of conversational thinking in a practical setting requires me to highlight some of its key features. The conversational method as a tool (see for example CHIMAKONAM 2015; 2017ab) acknowledges that "despite all of its progress [our world] is in a constant state of tension brewed by our failure to communicate across culture, ethnic, religious and epistemic borders and [to] understand ourselves as human beings with a common destiny" (CHIMAKONAM 2017a, 13). It is, therefore, particularly suited for practical application in cross-cultural exchanges, such as those between immigrant and host communities in many parts of Europe. It describes a relational, creative and critical procedure between two agents:

Conversationalism presupposes relationship(s) between 'nwa-nsa' who is the epistemic agent that created an idea and 
therefore shoulders the responsibility of defending, clarifying or revising it and 'nwa-nju', who can be defined as an epistemic agent that questions the viability and veracity of an idea created by nwa-nsa. This relationship is a critical one and may be described as a process of 'creative struggle'[...] (CHIMAKONAM 2017a, 17).

This creative struggle pans out as a process of sustained disagreement between agents in the interest of refining each thesis. In other words, the sustenance of an engagement is prioritized over its outcome and no synthesis is actively sought. Rather, each thesis is re-invented and grows in sophistication (CHIMAKONAM 2017a, 16). The rationale for this approach can be found in the assumption that truth value is contextual - or located within the context of propositions. These "can be evaluated true or false not, as we suppose, on the bases of the facts they assert but rather, on the bases of the contexts in which they assert those facts" (CHIMAKONAM 2017a, 25). Thus, conversationalism assesses validity from within a specific context or thought world. It eschews consensus with a view that a sustained collision of thesis and anti-thesis can produce greater philosophical rigor and sophistication.

However, as epistemic agents, we also live in an experienced (rather than merely theoretical) reality. So, the question becomes what happens when proponents of different/opposing theses take collective action on something they fundamentally disagree on? Collective action can be thought of as the action taken together by a group of people whose goal is to enhance their condition and achieve a common objective (CORREA 2020). There are many formulations and applications of this in the social sciences, such as psychology, sociology, anthropology, political science and economics (see VAN ZOMEREN, POSTMES \& SPEARS 2008). For the context of this paper, I suspend the idea of a unified or harmonized vision commonly informing notions of collective action (see CORREA 2020) and refer to it simply as action 'taken together' by a group of people - even as a result of compromise. As I will show, this reflects the experience of those who engaged in a conversational exchange about whether the hijab should be worn in educational settings in Austria. In the absence of consensus and, as epistemic agents refined and entrenched opposing theories, a compromise on the practicalities of this policy occurred. 
This relationship of practicality and theory, of thought and action, is explored through discourse theory, which posits that the way we think about things shapes our social reality and our social reality shapes the way we think about things (VAN DIJK 2001). This means that the discourses we produce are not just a reflection of the way things are. Rather, they are constructed and can be de- and reconstructed depending on our ethical or cultural lens. Such a lens relates to the concept of the 'Context-dependence of Value' (CHIMAKONAM 2017a, 2) or the idea that epistemic propositions hold true in specific contexts and from certain vantage points. For this paper, therefore, my attention is centered around analyzing how social practice (in the form of collective action) can affect, reshape and reposition that vantage point for various epistemic agents who are engaged in discursive processes such as the creative struggle.

\section{Account and Analysis}

\section{Background and Context}

The engagement summarized and reflected upon here took place over several weeks in the spring of 2019. It is embedded within a community initiative in one of Vienna's culturally diverse districts that aim to bring together people from Austrian and refugee backgrounds at a local center for cultural exchanges. The initiative encompasses many components, including German lessons and the study of materials that facilitate the integration of refugees into Austrian society. My specific involvement was an experiment within this greater initiative and was based on its founding (and decolonial) ideal that integration should not constitute the mere assimilation of 'other' cultures into a dominant one but rather that a more reciprocal and relational engagement could lead to a common, diversely textured and constantly evolving 'co-culture' - resembling the relational trajectory of conversationalism (see CHIMAKONAM 2017a).

Having just published a paper that had introduced me to conversational philosophy, I was curious to understand how its procedural elements might work. So, I invited four participants of the above initiative, two Austrian, one Afghani and one Iraqi (both refugees in Austria) to take part in a series of conversations with me to explore the hot topic of what policy they would design in order to 
address how hijabs should be handled in Austrian educational settings, such as schools or community centers. In each group, one participant identified as a woman and one as a man. Based on my own understanding, I then explained and encouraged us to follow a more or less conversational procedure (CHIMAKONAM 2015; 2017). This meant that, over several days, we worked on conceptualizing and developing distinct vantage points or theses through a rigorous process of engagement that refined and elevated each of our theses. A lot of effort was made to remain respectful and appreciative of others, even though a structurally argumentative approach took the place of explicitly consultative (KOLSTOE 1990) and deliberative (TAVERNARO-HAIDARIAN 2018) modes of conversation. This was done with a view to following the critical process of epistemic challenge and defense.

Within this engagement, I was both participant and observer, moderator and private individual with an affinity for multiculturalism. I was also the only one formally recording and reflecting on the experience. Although all participants engaged voluntarily and for the purpose of creating greater cultural understanding, I have anonymized the account by referring only to the proponents of each thesis as a group. This has been done to protect the identity of those whose refugee status remains volatile and because participant details are not relevant to the theoretical discussion at hand. Importantly, as none of us saw ourselves as formal philosophers or scholars of conversationalism, our understanding of, and experience with, the conversational method should be seen accordingly - namely as that of laywomen and men formulating laypeople 'theses'.

\section{Experience and Reflections}

Our first encounter began with us coming together in a park near the community center. My question to the group was: 'What should a policy on hijabs look like in Austrian educational settings?' Each group expressed their thoughts freely and without preparation or consultation. Conversations were informal rather than methodical and included references to the ways in which individuals had grown up, what values they held dear and how they saw the role of religion in society. Similarities between the views and thoughts of those who had grown up in Austria on the one hand and those who had grown up in 
Afghanistan and Iraq on the other soon began to crystalize. Two groups began to form along those lines.

It was during the second encounter that the process of reasoning and articulating a viewpoint became more systematic and began to shape into what could be called two distinct and internally coordinated 'theses' in the sense of positions and written down statements that declared what each group believed should be the policy and intended to persuade others of (see RORDBURG 1999). The initial thesis that emerged from the group comprising of two individuals who had grown up in Muslim societies outside of Austria/Europe (or group 1) became that 'Muslim women and girls are prescribed to and should wear a hijab in Austria because Godly law supersedes, and should ideally inform, all societal laws.' The initial thesis emerging from those who had grown up in Austria and experienced a Catholic and non-religious upbringing respectively (or group 2) became that 'Muslim women and girls in Austria should not wear a hijab because Austria is a secular state with a Catholic cultural heritage and living here requires accepting local norms the way foreigners living in a Muslim country have to.' In this conversational encounter then, the two propositions in themselves became thesis and anti-thesis, or nwa-nsa and nwa-nju as conversationalists like to say.

What followed was a series of more in-depth encounters in the park and at the community center over a span of several days. A process that had begun somewhat cautiously evolved into a more courageous and continuous procedure of testing and challenging, whereby each group questioned the epistemic strength of the arguments of the other with no express intention of finding a resolution. Without my explicit invitation, participants engaged in their own research and consulted other sources in an effort to defend, clarify, revise and strengthen their epistemic position vis-à-vis the other group. Moving away from what was initially an intuitive exchange, the discursive trajectory became more formal and structured with a legal emphasis. The resulting theses were thus refined to the following for group 1: 'Muslim women and girls are prescribed to and should wear a hijab in Austria because freedom of religion is a foundational principle of Austria law' and the following for group 2: Muslim women and girls in Austria should not wear a hijab because doing so threatens children's negative freedom of 
religion and state neutrality, which are also foundational principles of Austrian law'.

In order to gain new insights for the development of our theoretical positions, we decided to activate a policy that would pose a compromise for both parties and a policy that could be carried out within the walls of the community center. A mutual concession was formulated in the following way: 'Women can choose to wear the hijab, children and youth may not wear it'. Group 1 was challenged by this in that they felt that Muslim children and youth or the Muslim parents of children and youth should have a choice over whether they wanted their daughters to cover their hair, while group 2 felt that not even women should wear the hijab. However, the idea was that this compromise, once taken, would be actioned wholeheartedly and become the basis for further reflection and consideration down the line. Accordingly, the policy was carried out and tested by both groups and their peers in the community center for a period of two weeks. During this time, (parents of) girls who may have previously appeared with the hijab were kindly reminded to remove it. In the case of adults, we observed that only one woman who had previously come to the center wearing a hijab came without it. As it raises critical concerns about the gender dimensions of this 'conversation', I would like to include her reasoning here, which was expressed in the following way: 'The decision to wear the hijab or not should be held by women and only women. Laws that mandate or restrict it, strip that liberty from its rightful holder'. This was contrasted by views that religion, society or politics (including men) should be a part of this decision. During his time of action and exchange, the members of both groups engaged in many conversations with one another as well as with other women and men who frequented the center in order to explain the experiment and discuss its implications. These interactions were described by members of both groups as 'meaningful' and 'purposeful' and contributing to insights and the development of 'relationships' and sometimes 'veritable friendships' between themselves as well as between them and others with whom they were engaging and sometimes disagreed with.

Returning to the formal 'conversational' discussion after this period of time, I then asked members of both groups to use the insights they had gained through collective action to re-engage in the creative struggle of revisiting and refining their theses (which acted as thesis 
and anti-thesis). This was a little challenging as participants (including myself) felt somewhat disinclined to do so. When I asked if anyone could explain this sense of discomfort, one answer was formulated in this way: 'I feel like without talking about our positions we agree more but if we talk about it again, we are going to disagree again'. Collective action, then, had moved the experience from the realm of the intellectual into the realm of the personal, where deeper connections had been forged and a sense of harmony was created.

However, agreeing that disagreeing was actually desirable for the process of creative struggle and the purpose of this experiment, each group slowly returned to sharing their insights from the past two weeks. They articulated, challenged, revised and again refined their respective positions. The 'final' versions of their theses were then recorded as follows for group 1: 'Muslim women and girls are recommended (rather than prescribed) to wear a hijab but should do so of their own volition. Girls can be entirely exempt as they are not of age', and for group 2: 'Muslim women should have the freedom to choose to wear a hijab because diversity and choice are both a part of freedom. Girls should not be encouraged to wear the hijab because they are not old enough to make that choice for themselves but should also not be judged for wearing it. Discourse should occur on a cultural rather than legal level.' Both groups, then, had articulated final versions of their thoughts that were not the same but similar in effect. They emphasized freedom of choice and were more moderate in language because they replaced directives with recommendations. The following was then suggested as an actionable policy for the community center: 'Anyone can wear what they want.'

In order to summarize the above, I place upon this experience a retrospective structure, which consists of a phase of planning, action and reflection. Planning consists of articulating viewpoints and theses through creative struggle, action refers to taking collective action together and reflection means returning to the creative struggle to reflect on and refine each thesis. In summary, this is what the cycle of planning, action and reflection looked like: 
'What should a policy on hijabs look like in Austrian educational settings?'

\section{Planning}

A phase where two groups applied the conversational method to formulate theses:

Group 1: 'Muslim women and girls are prescribed to, and should, wear a hijab in Austria because Godly law supersedes, and should ideally inform, all societal laws'

VS

Group 2: 'Muslim women and girls in Austria should not wear a hijab because Austria is a secular state with a Catholic cultural heritage and living here requires accepting local norms the way foreigners living in a Muslim country have to'

In continuing with the conversational method, each group's theory grew in sophistication and stayed divergent:

Group 1: 'Muslim women and girls are prescribed to, and should, wear a hijab in Austria because freedom of religion is a foundational principle of Austrian law' VS

Group 2: 'Muslim women and girls in Austria should not wear a hijab because doing so threatens children's negative freedom of religion and state neutrality, which are also foundational principles of Austrian law'

\section{Action}

A practical policy was then decided on through compromise. It was actioned wholeheartedly:

'Women can choose to wear the hijab, children and youth should not wear it' 


\section{$\underline{\text { Reflection }}$}

After taking this collective action, participants returned to the conversational method to refine their theories:

$$
\begin{aligned}
& \text { Group 1: 'Muslim women and girls are } \\
& \text { recommended (rather than prescribed) to wear } \\
& \text { a hijab but should do so of their own volition. } \\
& \text { Girls can be entirely exempt as they are not of } \\
& \text { age' } \\
& \text { vs } \\
& \text { Group 2: 'Muslim women should have the } \\
& \text { freedom to choose to wear a hijab because } \\
& \text { diversity and choice are both a part of } \\
& \text { freedom. Girls should not be encouraged to } \\
& \text { wear the hijab because they are not old } \\
& \text { enough to make that choice for themselves but } \\
& \text { should also not be judged for wearing it. } \\
& \text { Discourse should occur on a cultural rather } \\
& \text { than legal level' }
\end{aligned}
$$

Renewed action

Participants unanimously agreed on a final policy:

'Anyone can wear what they want'

\section{Complications and Implications}

There are a number of possible objections or complications that could arise from my analysis. For example, in the absence of a more quantitative style of research, the subjectivity of my account can be seen as problematic. However, a subjective, interpretative approach is more suitable for exploring the distinctly 'human dimension' of research in which I am bound up here (CRESWELL 2013), and which relates to applying the conversational method. So, in lieu of any hard positivism, and by analyzing complex aspects of human culture, I 
fully embrace that there is no objective account of the world that I have direct access to. I, therefore, deliberately confer upon the data my own perspectival matrix and theoretical agenda (see GRAY, WILLIAMSON, KARP \& DALPHIN 2007). This agenda includes the proposition that while I (or any other individual or group) may not have access to an objective account, it might nonetheless exist. And that the practice of collaboration (in the form of collective action) may have brought us closer to it. Skeptics may argue, of course, that this collaboration may have occurred as a result of pressured acquiescence, which could be associated with collectivist tendencies. However, as no group was absorbed by the theoretical position of the other prior to compromising on an initial policy, and since neither group subsequently approached the other in any larger measure than the other, there is still enough evidence to suggest that it was in fact a shared experience of life or a shared 'context', which led to epistemic closeness - or complementarity, as conversationalists say.

The above experience, however modest in scope, then suggests that when those engaging in a conversational encounter accompany each other on a path of collective action related to their creative struggle, their understanding and conception of reality or 'truth' may begin to converge. In other words, by 'doing together', people may also end up 'thinking together' (and vice versa). As reflected in discourse theory, there is a symbiotic relationship between social reality and thought, language or discourse. The consequence is that, as long as our social reality remains divergent, so will our thoughts. The coming together of either thought or life-world, then, can bring about the coming together of the other. In the case of the above, the process of nurturing meaningful conversations while walking a path of action together began creating a new and common reality for the two groups of youth. They shared experiences and contexts, and this impacted the trajectory of their thoughts. Through this process, the relationship between each 'group' or 'community' was reconsidered. It moved from a concept of smaller, interconnected but separate locutions, towards a concept of overlapping or mutually inclusive ones. After all, it was 'together' that capacities and insights were gained, through a pattern of collective planning, action and reflection.

Theoretical implications of the above for the development of conversationalism begin with the idea that, considering the Context- 
dependence of Value (CHIMAKONAM 2017a, 2), contexts can be seen not only as relational to one another but also as fluid, sometimes overlapping, or indeed possibly converging in some way. The overall trajectory of conjunctive and disjunctive modes of thought, therefore, may still be moving towards a real or imagined point of intersection (CHIMAKONAM 2017b, 121). Moreover, while sustaining a continuous clash of differing opinions is desirable and leads to epistemic sophistication, the process of creative struggle need not categorically exclude a coming together of thought. The moderation, which was expressed in the final versions of the two theses, did not amount to epistemic diffusion. Rather, each thesis was epistemically enriched based on the practical experience that was gained and the subsequent reflection that occurred. In cultivating an exchange with those whom the hijab policy affected, first-hand information flowed back into the process of theory development, resulting in a renewed layer of nuance. Hence, sophistication did not take place exclusively through cultivating divergence but also by allowing convergence. Finally, and in considering also macro-theoretical implications, the idea of fluid and converging experiences and thought worlds contributes to alternative conceptions of the relationship between absolutism and relativism.

Thought of as separate categories, Western social theory often frames absolutism and relativism, or objectivism and subjectivism, as dichotomous (see SMITH \& KARLBERG 2009, 60). Truths are seen as either objective and absolute on the one hand, or contextual, subjective and relative on the other. But by considering the Contextdependence of Value as shifting and capable of convergence, this relationship can be rethought in terms of engendering an evolutionary trajectory. By this is meant the possibility of absolute or objective truth (even if only as a concept) towards which nested and contextual ones may gradually advance. In this way, simultaneity is further advanced, which produces new concepts and opens up new vistas for thought (CHIMAKONAM 2017, 15). In contributing to a procedure that transcends adversarial-colonial posturing in favor of a (critical yet) deeply complementary one, this reflects trends in decolonization that rely on relational strategies for social change (see TAVERNAROHAIDARIAN 2019). As one participant of my community conversational experiment said: 
By trying out the [conversational] method and then doing something about it together, we developed the capacity to have meaningful conversations. We built trust. We now dare to share unfinished thoughts and try to speak 'truth' amongst each other. 'My' truth, 'your' truth. And sometimes 'our' truth (Participant, group 2).

Practically and for macro-cultural exchanges between Austrians or Europeans and their immigrant population this can mean that groups of people can live out a rich and dynamic tapestry of identities and values, while nonetheless approaching one another under an overarching and shared sense of humanity.

\section{Conclusion}

Conversationalism seeks to make meaning through creative struggle (CHIMAKONAM 2021, 1). It is about harnessing a continuous collision of thesis and anti-thesis in a process that yields ever-higher levels of epistemic sophistication. Yet, theses, in and of themselves, are inconsequential if they begin in words and end in words. Testing them and allowing the insights gained from their application to feed back into their refinement opens up a possibility for learning. With this in mind, my paper sought to capture and reflect on the experience of a group of micro-intercultural conversationalists who applied the conversational method for the first time and without any formal background or training in philosophy. My account and analysis showed that when this group took collective action on the divergent epistemic positions they had developed, this shared experience created a contextual overlap and conceptual nearness while, nonetheless, helping them refine the theses they had articulated.

For the theory development of conversationalism, this suggests that the process of creative struggle can benefit from collective action and that convergence of thought or the collective birthing of something new is not always counter-productive. It may occur as a result of each thesis gaining higher ground. Hence, a new vision opens up for how epistemic contexts or thought worlds relate to one another. They can be seen as relative or subjective but also as capable of approaching one another or moving towards one objective and absolute 'truth' as they become more and more sophisticated. In 
furthering a procedure that transcends adversarial-colonial posturing in favor of a (critical yet) complementary one, this advances the project of decolonization and has practical implications for intercultural societal life, such as that emerging in many parts of Europe and elsewhere. For example, it is possible that groups of people can live out a rich and dynamic tapestry of identities and values, while nonetheless collaborating under an overarching and shared sense of humanity. Emerging from this study is also the opportunity to further research and reflect on processes of joint decision-making. In the experience shared above, there was no epistemic alignment as groups tried to agree on a collective path of action. It would be valuable, therefore, to take a closer look at the deliberative procedure that allowed proponents of different/opposing theses to reach a compromise as well as the role that wholeheartedly carrying out that decision (rather than sabotaging it) played for the subsequent project of returning to the creative struggle.

\section{RELEVANT LITERATURE}

1. CORREA, Gustavo. "Community and Collective Action: Adaption of a Talk given at the Bahá'í World Centre in 2015," N.P, September, 2020. Retrieved May, 2021.

2. CHIMAKONAM, Jonathan. O. "What, Really, is this Thing called Conversational Thinking?," [in this Issue].

3. "Conversationalism as Emerging Method of Thinking in and Beyond African Philosophy," [Acta Academia], pp11-33, December, 2017a. Vol 49. No 1.

4. "What Is Conversational Philosophy? A Prescription of a New Theory and Method of Philosophising, in and Beyond African Philosophy," [Phronimon], pp115-130, 2017b. Vol 18.

5. “_Conversational Philosophy as a New School of Thought in African Philosophy: A Conversation with Bruce Janz on the Concept of 'Philosophical Space'," [Confluence: Online Journal of World Philosophies], pp9-40, 2015. Vol 3.

6. CRESWELL, John. W. [Research Design: Qualitative, Quantitative and Mixed Method Approaches], 2013. Sage: London. 
7. ELLIS, Carolyn. [The ethnographic I: A Methodological Novel about Autoethnography], 2004. AltaMira Press: Walnut Creek, CA.

8. ELLIS, Carolyn., ADAMS, Tony., \& BOCHNER, Arthur. P. "Autoethnography: An Overview," [Forum: Qualitative Social Research], pp1-10, January 2011. Vol 12. No 1.

9. FOUCAULT, Michel. [Mental illness and psychology], 1987. University of California: Berkley.

10. GAVEY, Nicola. "Feminist Poststructuralism and Discourse Analysis Revisited," [Psychology of Women Quarterly], pp183-188, March 2011. Vol 35. No 1.

11. GRAY, Paul S., WILLIAMSON, John., KARP, David A., \& DALPHIN, John. R. [The Research Imagination: An Introduction to Qualitative and Quantitative Methods], 2007. Cambridge University Press: Cambridge. Paperback.

12. HERRMANN, Andrew. F. "My Father's Ghost: Interrogating Family Photos," [Journal of Loss and Trauma], pp337-346, February 2005. Vol 10. No 4.

13. KOLSTOE, John. E. [Consultation: A Universal Lamp of Guidance], 1990. George Ronald: Oxford. Paperback.

14. RODBURG, Maxine. [Developing a Thesis], 1999. Harvard University Press: Cambridge MA. Paperback.

15. SMITH, Todd., \& KARLBERG, Michael. "Articulating a Consultative Epistemology: Toward a Reconciliation of Truth and Relativism," [The Journal of Studies], pp59-99, December 2009, Vol 19. No 1.

16. TAVERNARO-HAIDARIAN, Leyla. "Decolonization and Development: Reimagining Key Concepts in Education," [Research in Education], pp19-33, May 2019. Vol 103. No 1.

17. "Deliberative Epistemology: Towards an Ubuntu-based Epistemology that Accounts for a Priori Knowledge and Objective Truth" [South African Journal of Philosophy], pp229-242, June 2018. Vol 37. No 2.

18. USSHER, Jane M. and PERZ, Janette. "Critical Discourse/Discourse Analysis," [Handbook of Research Methods in Health Social Sciences, Pranee LIAMPUTTONG Ed.], pp882-894, 2019. Springer Nature Singapore Pte. Ltd: Singapore. 
19. VAN DIJK, Teun, A. "Critical Discourse Analysis," [The Handbook of Discourse Analysis, Deborah SHIFFRIN, Deborah TANNEN \& Heidi E. Hamilton Eds.], pp352-372, 2001. Blackwell: Oxford.

20. VAN ZOMEREN, Martijn., POSTMES, Tom., \& SPEARS, Russell. "Toward an Integrative Social Identity Model of Collective Action: A Quantitative Research Synthesis of Three Socio-Psychological Perspectives," [Psychological Bulletin], pp504-535, August 2008. Vol 134. No 4.

21. WETHERELL, Margaret. "Positioning and Interpretative Repertoires: Conversation Analysis and Post-Structuralism in Dialogue," [Discourse and Society], pp387-412 July 1998. Vol 9. No 3.

22. WILLIG, Carla. [Introducing Qualitative Methods in Psychology: Adventures in Theory and Method], 2008. McGraw Hill: Maidenhead. 